http://dx.doi.org/10.22319/rmcp.v9i4.4654

Artículo

\title{
Análisis de pedigrí en la determinación de la diversidad genética de poblaciones bovinas para carne mexicanas
}

\section{Pedigree analysis for determination of genetic diversity in mexican beef cattle populations}

Rodolfo Ramírez-Valverde ${ }^{\mathrm{a}}$

Antonio R. Delgadillo-Zapata ${ }^{a}$

Joel Domínguez-Viveros ${ }^{\mathrm{b}}$

Jorge A. Hidalgo-Moreno ${ }^{\text {a }}$

Rafael Núñez-Domínguez ${ }^{\text {a* }}$

Felipe A. Rodríguez-Almeida ${ }^{\mathrm{b}}$

Claudia Reyes-Quiroz ${ }^{\mathrm{a}}$

José G. García-Muñiz

a Universidad Autónoma Chapingo. Departamento de Zootecnia.. Km 38.5 Carretera México-Texcoco. 56230, Chapingo, Estado de México. México.

${ }^{\mathrm{b}}$ Universidad Autónoma de Chihuahua. Facultad de Zootecnia y Ecología. Chihuahua, Chih. México.

*Autor de correspondencia: rafael.nunez@correo.chapingo.mx

\section{- Resumen:}

El objetivo de este estudio fue evaluar la diversidad genética de siete poblaciones de bovinos para carne en México, mediante análisis de sus pedigríes. Los análisis se realizaron con el 
programa Endog, utilizando información de los animales inscritos en el libro genealógico nacional de cada raza. Los tamaños de los pedigríes estudiados fueron: Angus $(A N)=73,271$; Brangus Negro $(B N)=68,474$; Brangus Rojo $(B R)=12,925$; Hereford $(\mathrm{HE})=13,248$; Limousin $(\mathrm{LI})=53,221$; Salers $(\mathrm{SA})=14,065$; y Suizo Europeo $(\mathrm{SE})=184,788$. En general, las poblaciones mostraron mejoras importantes en la integridad de sus pedigríes en los 10 años más recientes, con parámetros comparables a los publicados en otras poblaciones de bovinos. El intervalo generacional varió de 5.1 a 7.2 años entre razas. Los coeficientes de consanguinidad y de relación genética aditiva promedio fueron relativamente bajos en las diferentes poblaciones (0.9-4.2 y 0.3-6.5\%, respectivamente); aunque en algunas razas (BN, BR y HE) se detectaron incrementos de estos indicadores en los 10 años más recientes. El tamaño efectivo de población fluctuó entre 24 y 192, con valores menores que 50 en BR y SA. Los parámetros relacionados con la probabilidad de origen de los genes indican que las contribuciones desbalanceadas de los animales fundadores, los cuellos de botella y la deriva genética, han tenido un efecto importante en las poblaciones actuales, con la consecuente pérdida de diversidad genética. Se recomienda continuar o adoptar estrategias de apareamiento que minimicen la consanguinidad, y el uso intensivo de pocos animales para mantener la variabilidad genética de futuras generaciones, así como ampliar la diversidad genética mediante el uso estratégico de genes provenientes de otros países.

- Palabras clave: Tamaño población, Consanguinidad, Número ancestros, Intervalo generacional.

\section{- Abstract:}

The objective of this study was to evaluate genetic diversity of seven Mexican beef cattle populations, using pedigree analyses. The analyses were carried out with the Endog software, using information of the registered animals on national herd-book of each breed. The size of the populations studied were: Angus $(A N)=73,271$; Black Brangus $(B N)=68,474$; Red Brangus $(B R)=12,925$; Hereford $(\mathrm{HE})=13,248$; Limousin $(\mathrm{LI})=53,221$; Salers $(\mathrm{SA})=$ 14,065; and Braunvieh $(\mathrm{SE})=184,788$. In general, the populations showed important improvements in pedigree integrities for the more recent $10 \mathrm{yr}$, with comparative parameters to other published in cattle populations. The generation interval varied among breeds from 5.1 to $7.2 \mathrm{yr}$. The average inbreeding and relatedness coefficients were relatively low in the different populations (0.9-4.2 and 0.3-6.5\%, respectively); although increases of these indicators during the 10 more recent years were detected in some breeds (BN, BR and HE). The effective population size of studied populations fluctuated between 24 and 192, with values lower than 50 in BR and SA. The parameters related with probability of gene origin indicated that the unbalanced contribution of founders, the bottlenecks and genetic drift have had an important effect on the current populations with the consequent losses in genetic diversity. It is recommended to continue or adopt mating strategies that minimize inbreeding 
and the intensive use of few animals to maintain genetic variability in future generations, and to increase the genetic diversity through the strategical use of genes from other countries.

- Key words: Population size, Inbreeding, Number ancestors, Generation interval.

Recibido 04/10/2017

Aceptado 26/12/2017

\section{N Introducción}

El monitoreo de la estructura poblacional, la consanguinidad, el tamaño efectivo de población y la probabilidad de origen de los genes, permite prevenir pérdidas de diversidad genética en las poblaciones bovinas ${ }^{(1)}$, particularmente en poblaciones bajo procesos de selección ${ }^{(2)}$. Por lo anterior, su estudio es necesario para implementar de manera más integral los programas de selección ${ }^{(3)}$.

En varios países se ha generado información acerca de la diversidad genética de sus poblaciones animales, tanto para razas locales como para transfronterizas (presentes en varios países), lo que ha sido ampliamente estudiado mediante análisis de pedigrí y usado en poblaciones de animales domésticos ${ }^{(1-5)}$. Sin embargo, en México, los estudios publicados utilizando el análisis de pedigrí en sus poblaciones han sido escasos ${ }^{(6,7)}$, por lo que el análisis de la estructura y variabilidad genética de sus poblaciones de animales domésticos debe ser una acción prioritaria de investigación en mejoramiento genético animal ${ }^{(8)}$.

Los métodos de selección comúnmente usados, como los basados en las predicciones de los valores genéticos obtenidos mediante el modelo animal, pueden incrementar los niveles de consanguinidad y disminuir la variabilidad genética de las poblaciones ${ }^{(9)}$. En México, las evaluaciones genéticas nacionales en razas de bovinos para carne iniciaron en 2001. En 2010 se reportó que 21 razas ya tenían evaluaciones genéticas ${ }^{(10)}$, en las cuales es importante conocer la diversidad genética presente. Por lo anterior, el objetivo de este estudio fue evaluar la diversidad genética de siete poblaciones de bovinos para carne en México, mediante el análisis de sus pedigríes. 


\section{Material y métodos V}

La información genealógica utilizada fueron las bases de datos de las poblaciones nacionales de bovinos Angus (AN), Brangus Negro (BN), Brangus Rojo (BR), Hereford (HE), Limousin (LI), Salers (SA) y Suizo Europeo (SE); provenientes de las respectivas asociaciones mexicanas de criadores de ganado de registro. El libro genealógico de cada raza incluyó animales nacidos en los periodos 1973-2017, 1964-2016, 1973-2017, 1935-2016, 1953-2017, 1976-2016 y 1927-2016 para AN, BN, BR, HE, LI, SA y SE, respectivamente. Los pedigríes originales se editaron para evitar la presencia de incongruencias, como registros duplicados, edades de los hijos mayores que las de sus progenitores o diferencias de edad no razonables biológicamente, y registros de animales como padres y como madres.

Los análisis de los pedigríes se llevaron a cabo mediante el programa Endog v4.8, donde se describen los detalles de los procedimientos usados para estimar los parámetros poblacionales estudiados ${ }^{(11)}$. Los análisis se realizaron para el total de los animales (PT) en cada raza, y para sus poblaciones de referencia (PR) se consideraron los animales en los 10 años más recientes (potencialmente vivos) de la población correspondiente. El significado de los parámetros poblacionales se describe a continuación.

\section{- Integridad del pedigrí •}

Los principales factores que influyen en la exactitud de los parámetros de las poblaciones usando el análisis de pedigrí son la integridad y nivel de completitud del pedigrí ${ }^{(12)}$. En este estudio, la integridad de los pedigríes se determinó mediante las estimaciones del número de generaciones completas $(G C)$, número máximo de generaciones trazadas $(M G T)$ y número de generaciones completas equivalentes $(G E)$. Se consideró una generación completa cuando se conocían los $2^{n}$ antepasados de un individuo, mientras que las generaciones máximas trazadas relacionaron el número máximo de éstas que separan a un individuo de su ancestro más lejano ${ }^{(11)}$. Para un individuo dado, el número de generaciones completas equivalentes se obtuvo mediante la suma para todos los ancestros conocidos de los términos calculados como 
la suma de $(1 / 2)^{\mathrm{n}}$ de todos los antepasados conocidos, donde $n$ es el número de generaciones que separan al individuo de cada ancestro conocido $^{(13)}$. Adicionalmente, como medida complementaria a la de integridad de los pedigríes, se calcularon los porcentajes de ancestros conocidos hasta la tercera generación parental.

\section{- Intervalo generacional (IG) •}

El $I G$ fue definido como la edad promedio de los padres cuando nace su descendencia ${ }^{(14)}$. Este parámetro se calculó para las cuatro rutas gaméticas de selección: padre-hijo, padre-hija, madre-hijo y madre-hija.

\section{- Coeficientes de consanguinidad (F) y de relación genética aditiva promedio (CRP) •}

El $F$ se calculó usando el algoritmo establecido por Meuwissen y Luo ${ }^{(15)}$, basado en el principio de que los fundadores no están emparentados y, además, en que su $F$ es igual a cero. El $C R P$ fue definido como la probabilidad de que un alelo escogido al azar de entre toda la población en el árbol genealógico pertenezca a un animal determinado ${ }^{(11)}$; por tanto, es una medida del porcentaje de genes que, en promedio, comparte cada individuo con el resto de los individuos del pedigrí. El cálculo del CRP se obtuvo del promedio de los coeficientes en la fila correspondiente al individuo en la matriz de relaciones genéticas aditivas " $\mathrm{A}$ "(14,16). 


\section{- Tamaño efectivo de población $\left(\mathrm{N}_{\mathrm{e}}\right)$ y tasa de incremento en consanguinidad $(\Delta \mathrm{F}) \bullet$}

El $N_{e}$ es el número de individuos que darían lugar a la tasa calculada de consanguinidad, si fueran criados bajo el sistema de la población idealizada ${ }^{(17)}$. El $N_{e}$ representa las relaciones de parentesco entre los números de machos y hembras que contribuyen genéticamente en la población y el parámetro es inversamente proporcional a la $\Delta F^{(5)}$. El incremento en consanguinidad se estimó mediante: $\Delta F_{i}=1-\sqrt[t]{1-F_{i}}$, donde $t$ es el número de generaciones equivalentes. El $N_{e}$ se basó en el $\Delta F_{i}$ y se calculó promediando los $\Delta F_{i^{\prime} s}$ de los $n$ individuos incluidos como ${\overline{N_{e}}}_{e} \frac{1}{2 \overline{\Delta F}}{ }^{(18,19)}$. Ambos indicadores $\left(\Delta F\right.$ y $\left.N_{e}\right)$ fueron estimados para las PR respectivas.

\section{- Probabilidad de origen de los genes •}

El pedigrí de un individuo puede ser rastreado para calcular la contribución a su genoma de su eventual fundador o ancestro. Un fundador fue definido como un ancestro con progenitores desconocidos, pero cuando sólo se conoce un progenitor, el progenitor desconocido es considerado como fundador. La preservación de la diversidad genética de los fundadores hacia la población de estudio puede ser medida por sus contribuciones ${ }^{(12)}$.

Se consideró como número efectivo de fundadores $\left(f_{e}\right)$ al número de animales fundadores que contribuyeron por igual para producir la diversidad genética observada en la población $^{(20)}$. Si todos los fundadores contribuyen por igual a la descendencia, $f_{e}$ es igual al número de fundadores $(f)$; por el contrario, si la contribución es desigual, $f_{e}$ es de menor valor $^{(12,20)}$. Por otro lado, se consideró como número efectivo de ancestros $\left(f_{a}\right)$ al número mínimo de ancestros (fundadores o no) necesarios para explicar la diversidad genética completa de una población ${ }^{(12)}$. Este parámetro toma en cuenta la posible existencia de cuellos de botella en el pedigrí, por el uso extensivo de algunos individuos en ciertas generaciones. Finalmente, se consideró el parámetro número efectivo de genomas fundadores equivalentes $\left(f_{g}\right)$ al número de fundadores que podría esperarse produjeran la misma diversidad genética de la población bajo estudio, si los fundadores estuvieran igualmente representados y no 
hubiera ocurrido pérdida de alelos ${ }^{(21)}$. Adicionalmente, considerando las contribuciones genéticas marginales ${ }^{(12)}$, se obtuvieron los números de ancestros que explican el $50 \%$ de los genes, y la contribución del principal ancestro.

\section{| Resultados y discusión |}

\section{- Censos en las poblaciones •}

En el Cuadro 1 se muestra una descripción general de los animales considerados en cada raza estudiada. El número de animales utilizados por raza en el total del pedigrí fue variable (aproximadamente entre 13,000 y 185,000 para las PT, y entre 4,000 y 37,000 para las PR). Los promedios de hijos por semental en cada población fluctuaron entre 6 y 31, pero en las PR los valores fueron prácticamente el doble que en las respectivas PT, excepto en la raza SE que fueron la mitad. Los promedios de hijos por vaca oscilaron entre 1.8 y 2.7. Los porcentajes de animales con padre o madre desconocidos en las PT fue similar en todas las poblaciones, y en promedio fue alrededor de $16 \%$, oscilando para las diferentes poblaciones entre $8(\mathrm{BN})$ y $33 \%(\mathrm{HE})$; los valores disminuyeron sustancialmente para los animales nacidos en los 10 años más recientes (PR). 
Cuadro 1: Descripción general del número $(\mathrm{N})$ de animales $(\mathrm{A})$ en los pedigríes de las siete poblaciones bovinas mexicanas estudiadas, para la población total y la población de referencia* (dentro de paréntesis)

\begin{tabular}{lccccccc}
\hline & \multicolumn{7}{c}{ Población total (población de referencia) } \\
\cline { 2 - 7 } Concepto & AN & BN & BR & HE & LI & SA & SE \\
\hline NA total & 73,271 & 68,474 & 12,925 & 13,248 & 53,221 & 14,065 & 184,788 \\
& $(34,433)$ & $(30,356)$ & $(6,329)$ & $(4,444)$ & $(22,804)$ & $(4,885)$ & $(37,245)$ \\
N de sementales & 8,224 & 4,408 & 1,842 & 1,408 & 4,220 & 1,382 & 5,157 \\
& $(905)$ & $(696)$ & $(280)$ & $(123)$ & $(385)$ & $(67)$ & $(481)$ \\
N de madres & 30,948 & 25,090 & 6,124 & 4,587 & 20,587 & 5,377 & 59,609 \\
& $(8,357)$ & $(5,735)$ & $(1,895)$ & $(890)$ & $(4,067)$ & $(511)$ & $(5,042)$ \\
NA con padre & 10,539 & 5,612 & 2,133 & 4,413 & 6,219 & 1,768 & 21,845 \\
desconocido & $(988)$ & $(204)$ & $(5)$ & $(168)$ & $(213)$ & $(7)$ & $(3)$ \\
NA con madre & 11,420 & 5,048 & 2,129 & 4,450 & 6,812 & 1,765 & 24,337 \\
desconocida & $(1,282)$ & $(333)$ & $(9)$ & $(164)$ & $(389)$ & $(5)$ & $(4)$ \\
N de hijos por & 7.63 & 14.26 & 5.86 & 6.27 & 11.14 & 8.90 & 31.60 \\
semental (NHS) & $(16.67)$ & $(17.72)$ & $(10.90)$ & $(14.40)$ & $(20.87)$ & $(17.91)$ & $(16.36)$ \\
Máximo NHS & 412 & 561 & 312 & 150 & 710 & 358 & 1,620 \\
& $(246)$ & $(293)$ & $(219)$ & $(71)$ & $(182)$ & $(65)$ & $(155)$ \\
N de hijos por vaca & 2.00 & 2.53 & 1.76 & 1.92 & 2.25 & 2.29 & 2.70 \\
& $(2.00)$ & $(1.97)$ & $(1.80)$ & $(1.92)$ & $(2.14)$ & $(1.97)$ & $(1.78)$ \\
N de hatos & 202 & 152 & 86 & 48 & 214 & 118 & 648 \\
& $(175)$ & $(122)$ & $(70)$ & $(37)$ & $(132)$ & $(21)$ & $(308)$ \\
\hline
\end{tabular}

AN= Angus; BN= Brangus Negro; BR= Brangus Rojo; HE= Hereford; LI= Limousin; SA= Salers; SE= Suizo Europeo.

* Población de referencia (10 años más recientes).

\section{- Integridad de los pedigríes •}

Los valores promedio de los $G C, M G T$ y $G E$ para las poblaciones bovinas estudiadas se muestran en el Cuadro 2. De acuerdo con los $G E$ obtenidos para la PT, las poblaciones con mayor información sobre las genealogías fueron $\mathrm{BN}$ y $\mathrm{SE}(G E>4)$, mientras que en las demás poblaciones, los valores estimados (2.0 a 3.9) fueron inferiores a los obtenidos en otras investigaciones con razas europeas transfronterizas para carne $(4.0 \text { a } 9.3)^{(22,23)}$. Los valores para los $M G T$ y $G C$ en las razas estudiadas tuvieron un patrón similar al observado para los $G E$. Con excepción de HE, los $M G T$ estimados para las demás poblaciones fueron superiores o similares (5.4 a 10.3) a los estimados (4.2 a 6.9) ${ }^{(24,25)}$ en razas bovinas criollas (Costeño con Cuernos, Blanco Orejinegro, Romosinuano y Sanmartinero) y transfronterizas (Guzerat). Sin embargo, inferiores $(12 \text { a } 14)^{(22)}$ a los publicados para otras razas bovinas transfronterizas 
(Chianina, Marchigniana y Romagnola). Los $G C$ estimados en las poblaciones (2.4 a 3.0) fueron mayores que los obtenidos en bovinos Guzerat $(1.7)^{(25)}$, con excepción de la población HE, donde se obtuvo un valor menor (1.5). Los GE, GC y $M G T$ obtenidos en la PR fueron mayores que los de las respectivas PT y comparables con los publicados en otras poblaciones de bovinos ${ }^{(22-25)}$.

Cuadro 2: Números de generaciones máximas trazadas $(M G T)$, completas $(G C)$ y completas equivalentes $(G E)$ conocidas en los pedigríes de las siete poblaciones bovinas mexicanas estudiadas, para la población total y la población de referencia* (dentro de paréntesis)

\begin{tabular}{lccccccc}
\hline & \multicolumn{7}{c}{ Población total (población de referencia) } \\
\cline { 2 - 8 } Indicador & AN & BN & BR & HE & LI & SA & SE \\
\hline MGT & $8.09(11.61)$ & $10.28(13.48)$ & $5.40(8.09)$ & $3.32(6.22)$ & $7.65(11.16)$ & $6.83(10.61)$ & $8.50(13.03)$ \\
GC & $2.52(3.41)$ & $3.05(3.66)$ & $2.29(3.25)$ & $1.45(2.55)$ & $2.41(3.50)$ & $2.58(3.93)$ & $2.37(3.64)$ \\
GE & $3.87(5.32)$ & $5.89(7.51)$ & $3.38(4.91)$ & $2.03(3.55)$ & $3.76(5.42)$ & $3.84(5.62)$ & $4.28(6.38)$ \\
\hline
\end{tabular}

AN= Angus; BN= Brangus Negro; BR= Brangus Rojo; HE= Hereford; LI= Limousin; SA= Salers; SE= Suizo Europeo.

* Población de referencia (10 años más recientes).

Según Maignel et al ${ }^{(26)}$, el criterio más apropiado para caracterizar la información del pedigrí es el $G E$, mientras que los $M G T$ y $G E$ son indicadores complementarios que dan mejor idea del grado de profundidad del pedigrí. Las proporciones de ancestros conocidos sobre todas las generaciones trazadas $(G E)$ obtenidas en este estudio para las PT, indican que algunas estimaciones de parámetros poblacionales pudieran tener limitaciones al compararse con las de otras poblaciones de otros estudios. Sin embargo, con excepción de HE, para las PR de las demás poblaciones estos valores fueron mayores (4.9 a 7.5), lo que muestra los esfuerzos recientes de los criadores en el registro de información genealógica de sus animales.

El grado de integridad de los pedigríes en las poblaciones estudiadas se confirmó con las estimaciones de porcentajes de ancestros conocidos (Cuadro 3). La PT de HE presentó los menores porcentajes de progenitores conocidos hasta la tercera generación (38 a $67 \%$ ); mientras que las demás poblaciones presentaron porcentajes razonables de ancestros conocidos en la primera $(>84 \%)$, segunda $(>71 \%)$ y tercera $(>61 \%)$ generación parental. En las PR, los porcentajes de progenitores conocidos hasta la tercera generación aumentaron sustancialmente (>90\%), con excepción de HE, donde los valores fueron entre 77 y $90 \%$. Los porcentajes de ancestros conocidos publicados en otros estudios han sido variables; valores superiores al $90 \%$ de ancestros conocidos en la tercera generación parental han sido reportados para la población Marchigiana ${ }^{(27)}$, mientras que en las poblaciones españolas Bruna de los Pirineos y Asturiana de los Valles se han estimado valores cercanos al $3 \%{ }^{(14)}$. Por otro lado, las poblaciones de este estudio mostraron un patrón similar de valores entre las rutas paterna y materna, lo que sugiere que la captura de registros fue equitativa entre 
sexos. Conocer la profundidad del pedigrí es importante, ya que esto influye en la estimación de parámetros que son sensibles a la completitud de la información genealógica disponible $^{(12)}$; por lo anterior, para HE se esperarían subestimaciones en algunos de los parámetros analizados, principalmente en los $F$.

Cuadro 3: Porcentajes de ancestros conocidos de las siete poblaciones bovinas mexicanas estudiadas, para la población total y la población de referencia* (dentro de paréntesis)

\begin{tabular}{lccccccc}
\hline & \multicolumn{7}{c}{ Población total (población de referencia) } \\
\cline { 2 - 8 } Ancestros $^{*}$ & AN & BN & BR & HE & LI & SA & SE \\
\hline Progenitores & & & & & & & \\
S & $86(98)$ & $92(100)$ & $84(100)$ & $67(98)$ & $88(99)$ & $87(100)$ & $88(100)$ \\
M & $84(97)$ & $93(100)$ & $84(99)$ & $66(98)$ & $87(99)$ & $87(100)$ & $87(100)$ \\
Abuelos & & & & & & & \\
SS & $80(98)$ & $90(99)$ & $76(99)$ & $56(97)$ & $84(99)$ & $82(100)$ & $84(100)$ \\
MS & $80(96)$ & $89(99)$ & $75(97)$ & $56(97)$ & $84(99)$ & $80(100)$ & $82(100)$ \\
SM & $74(95)$ & $86(97)$ & $71(98)$ & $49(88)$ & $77(97)$ & $78(100)$ & $78(100)$ \\
MM & $73(93)$ & $87(98)$ & $71(98)$ & $49(88)$ & $76(96)$ & $78(100)$ & $77(100)$ \\
Bisabuelos & & & & & & & \\
SSS & $75(96)$ & $87(99)$ & $69(99)$ & $46(90)$ & $81(99)$ & $78(100)$ & $82(100)$ \\
MSS & $76(97)$ & $86(97)$ & $69(97)$ & $46(90)$ & $81(97)$ & $72(100)$ & $77(98)$ \\
SMS & $72(96)$ & $84(96)$ & $65(96)$ & $44(91)$ & $76(99)$ & $71(94)$ & $77(98)$ \\
MMS & $73(96)$ & $84(96)$ & $66(96)$ & $44(90)$ & $76(89)$ & $72(98)$ & $74(99)$ \\
SSM & $69(94)$ & $84(97)$ & $65(97)$ & $40(82)$ & $73(97)$ & $74(100)$ & $74(99)$ \\
MSM & $69(95)$ & $82(95)$ & $65(96)$ & $39(82)$ & $72(96)$ & $74(99)$ & $72(100)$ \\
SMM & $64(92)$ & $81(96)$ & $61(96)$ & $38(77)$ & $64(95)$ & $70(98)$ & $66(99)$ \\
MMM & $63(90)$ & $82(96)$ & $61(96)$ & $38(77)$ & $64(88)$ & $71(100)$ & $63(98)$ \\
\hline
\end{tabular}

$A N=$ Angus; $B N=$ Brangus Negro; $B R=$ Brangus Rojo; $H E=$ Hereford; $L I=$ Limousin; $S A=$ Salers; $S E=$ Suizo Europeo.

* Población de referencia (10 años más recientes).

${ }^{\mathrm{S}} \mathrm{S}=$ semental; $\mathrm{M}=$ madre.

\section{- Intervalo generacional}

Los $I G$ estimados para las poblaciones estudiadas en las cuatro rutas de selección posibles se muestran en el Cuadro 4. En promedio, para las PT los $I G$ de las razas estudiadas fluctuaron entre 5.1 y 6.5 años, incrementándose sus valores en todas las PR (5.5 a 7.2 años). Las 
pérdidas de diversidad genética se producen a mayor velocidad con menores $I G$; sin embargo, provocan mayor progreso genético por unidad de tiempo ${ }^{(16)}$. En general, los $I G$ promedios de las poblaciones de este estudio fueron menores a los de otros estudios ${ }^{(25,28,29)}$ con bovinos para carne de razas transfronterizas (Guzerat, Charolais, Limousin, Hereford, Angus y Simmental) y locales (Alentejana). Sin embargo, para LI y SE en la PT, y para las PR, los $I G$ fueron mayores en la ruta de padres para producir hijos que en las demás rutas gaméticas, lo que sugiere el uso de sementales por periodos prolongados, con posiblemente un uso de pocos reproductores e inseminación artificial. Este patrón de comportamiento con mayores $I G$ para la ruta padre-hijo fue similar al publicado en Angus ${ }^{(28)}$ y Suizo Pardo ${ }^{(30)}$. En investigaciones con razas bovinas locales, algunos estudios ${ }^{(14,24)}$ han estimado $I G$ en las rutas paternas menores que los de las maternas, atribuyéndolo al reemplazo temprano de los sementales.

Cuadro 4: Intervalos generacionales (años) estimados para las cuatro rutas gaméticas de selección en animales reproductores de las siete poblaciones bovinas mexicanas estudiadas, para la población total y la población de referencia* (dentro de paréntesis)

\begin{tabular}{lccccccc}
\hline & \multicolumn{7}{c}{ Población total (población de referencia) } \\
\cline { 2 - 8 } Ruta gamética & AN & BN & BR & HE & LI & SA & SE \\
\hline Padre-hijo & $6.19(6.64)$ & $5.25(5.82)$ & $5.54(6.50)$ & $4.93(6.71)$ & $7.67(11.12)$ & $5.09(7.57)$ & $8.19(8.90)$ \\
Padre-hija & $5.41(5.71)$ & $5.50(5.71)$ & $5.56(5.57)$ & $5.36(5.59)$ & $7.45(8.23)$ & $4.98(6.92)$ & $5.79(6.99)$ \\
Madre-hijo & $5.19(5.55)$ & $5.35(5.53)$ & $5.26(5.51)$ & $5.31(5.85)$ & $5.91(6.09)$ & $5.04(6.86)$ & $6.95(6.38)$ \\
Madre-hija & $5.27(5.59)$ & $5.74(5.82)$ & $5.14(5.21)$ & $5.45(5.54)$ & $5.50(5.63)$ & $5.19(6.71)$ & $5.95(6.40)$ \\
\hline Promedio & $5.4(5.7)$ & $5.6(5.8)$ & $5.3(5.5)$ & $5.4(5.7)$ & $6.5(7.2)$ & $5.1(6.9)$ & $6.4(6.7)$ \\
\hline
\end{tabular}

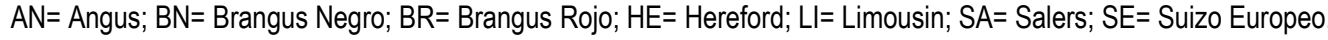

* Población de referencia (10 años más recientes).

Pirchner ${ }^{(31)}$ menciona que los $I G$ en las rutas padre-hijo= 6.5 a 9.0, padre-hija $=4.5$ a 6.5 , madre-hijo $=5.0$ a 6.5 y madre-hija $=5.0$ a 6.5 años, son valores que pueden considerarse como estándar en bovinos para carne. De acuerdo con esa clasificación, en general, los valores estimados en este estudio fluctuaron en esos rangos (con excepción de la PR de LI para las rutas padre-hijo y padre-hija, donde se obtuvieron $I G$ mayores a los estándares); sin embargo, para BN, BR, HE y SA en la ruta padre-hijo fueron inferiores a los estándares ( $<5.6$ años) en la PT, lo que sugiere recambios cortos y continuos de los sementales activos. 


\section{- Consanguinidad y relación genética aditiva promedio}

Los $F$ y los $C R P$ en las poblaciones estudiadas se muestran en el Cuadro 5. Los valores promedio de los $F$ para las PT de las poblaciones estudiadas fueron relativamente bajos ( 0.9 a $3.5 \%$ ), por lo que podría esperarse poca manifestación de la depresión endogámica; sin embargo, en los años recientes algunas poblaciones muestran mayores niveles de $F$ (>4 \%; BN y BR), posiblemente debido al uso más intensivo de pocos sementales. Resultados intermedios a los valores de $F$ obtenidos en este estudio han sido publicados para poblaciones de bovinos Tropicarne $\left(1.8 \%\right.$ y Suizo Europeo $(1.5 \%)$ de México ${ }^{(6)}$, y en varias poblaciones pequeñas de bovinos (Alistana, Morucha, Pirenaica, Blanco Orejinegro, Romosinuano, Marchigniana, Bonsmara, Charolais, Limousin y Simmental, entre otras) reportadas por diversos autores ${ }^{(14,24,27,32)}$. Los aumentos importantes en el porcentaje de animales consanguíneos y su consanguinidad promedio en la PR con relación a la PT, para las razas BN y SA, sugieren poca atención en la asignación de apareamientos de estas poblaciones en los años recientes.

Cuadro 5: Porcentajes de animales consanguíneos (PAF), del coeficiente de relación genética aditiva promedio $(C R P)$, y del promedio de coeficientes de consanguinidad en la población ( $F$-PO) y en los animales consanguíneos

$(F-$ AC) de las siete poblaciones bovinas mexicanas estudiadas, para la población total y la población de referencia* (dentro de paréntesis)

\begin{tabular}{lccccccc}
\hline & \multicolumn{7}{c}{ Población total (población de referencia) } \\
\cline { 2 - 8 } & AN & BN & BR & HE & LI & SA & SE \\
\hline PAF & $47.4(75.8)$ & $77.2(94.9)$ & $38.5(60.4)$ & $13.7(30.9)$ & $29.3(44.9)$ & $39.2(71.4)$ & $56.3(95.7)$ \\
F-PO & $0.9(1.4)$ & $3.5(4.8)$ & $2.6(4.0)$ & $0.9(1.8)$ & $0.9(0.8)$ & $0.9(2.2)$ & $1.5(2.4)$ \\
F-AC & $2.0(1.9)$ & $4.6(5.0)$ & $6.8(6.6)$ & $6.2(5.7)$ & $2.9(0.8)$ & $2.3(3.1)$ & $2.7(2.5)$ \\
CRP & $0.5(0.7)$ & $5.2(6.5)$ & $1.7(2.7)$ & $0.3(0.5)$ & $0.5(1.1)$ & $1.0(1.5)$ & $1.1(1.4)$ \\
\hline
\end{tabular}

$A N=$ Angus; $B N=$ Brangus Negro; $B R=$ Brangus Rojo; $H E=$ Hereford; $L I=$ Limousin; $S A=$ Salers; $S E=$ Suizo Europeo.

* Población de referencia (10 años más recientes).

Considerando los valores de todos los parámetros asociados con los $F$, las poblaciones $\mathrm{BN}$ y HE pudieran presentar problemas futuros, esta última debido a posibles subestimaciones en los parámetros, ya que tiene las mayores proporciones de padres y madres desconocidos (Cuadro 1), y menores integridades de sus pedigríes (Cuadros 2 y 3 ). 
Con la excepción de $\mathrm{BN}$ y $\mathrm{BR}$, los $C R P$ de las demás poblaciones fueron bajos $(<1.5 \%)$, lo que sugiere que la consanguinidad podría decrecer en las poblaciones si se practica migración entre hatos ${ }^{(14)}$ dentro de poblaciones. Los CRP estimados en BN y BR (>1.7 \%) sugiere problemas futuros para evitar apareamientos entre individuos no emparentados en esas poblaciones. Los $C R P$ son indicadores de la consanguinidad a largo plazo en una población; cuando estos alcanzan valores mayores que los $F$, los apareamientos deben ser cuidadosamente planificados, para evitar apareamientos entre animales emparentados ${ }^{(33)}$ y sugieren problemas de la población para mantener la variabilidad genética ${ }^{(34)}$, como es el caso de $\mathrm{BN}$ del presente estudio. $\mathrm{Al}$ respecto, Gutiérrez et $a l^{(14)}$ mencionan que un $C R P$ bajo junto con un $F$ alto en poblaciones, indica que la proporción de apareamientos dentro de hatos es alta y que el intercambio de animales entre hatos es poco frecuente. Una alternativa sugerida $^{(35)}$ es que los reproductores con un menor valor individual del $C R P$ deben preferirse como progenitores de las próximas generaciones, para minimizar los incrementos de los $F$ de los animales en el largo plazo.

La selección de animales basada en el mejor predictor linear insesgado (BLUP) también favorece la selección de animales emparentados y, por tanto, da lugar a valores altos de $F$ y $C R P^{(36)}$. En todas las poblaciones del presente estudio, los porcentajes de animales consanguíneos y los $F$ a través del tiempo mostraron una tendencia gradual creciente (Figura 1); además, la implementación de evaluaciones genéticas en las poblaciones estudiadas, acicatea la necesidad de establecer estrategias para el control de la consanguinidad. En los 10 años más recientes, aunque en forma fluctuante para algunas razas, la tendencia fue de mantener $\operatorname{los} F$, con la excepción de SA donde continuó el incremento gradual de $F$. Lo anterior sugiere atención de algunos criadores en la asignación de los apareamientos en los años recientes. 
Figura 1: Coeficientes de consanguinidad promedio (\%) de las siete poblaciones bovinas mexicanas estudiadas

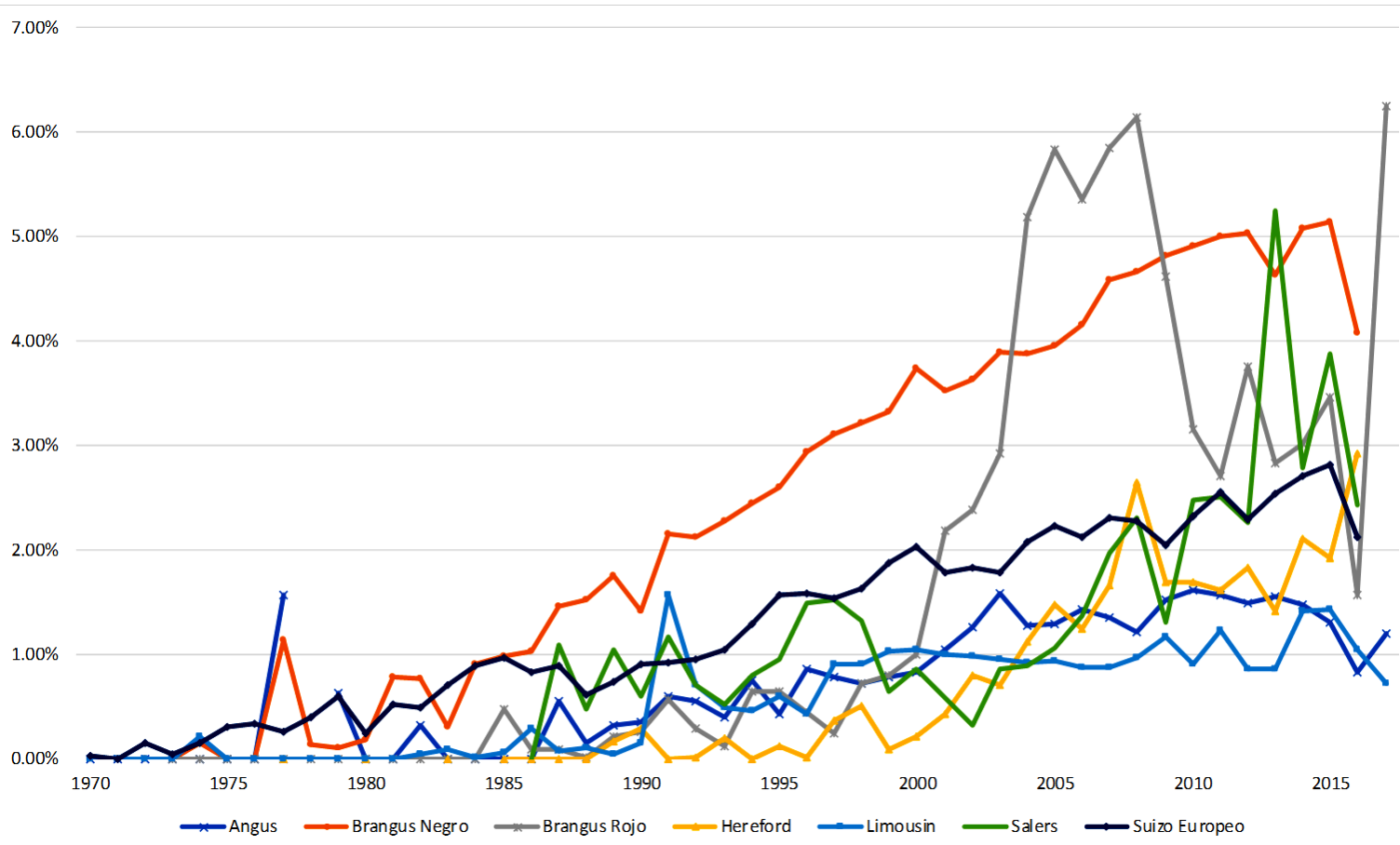

\section{- Tasa de incremento en consanguinidad y tamaño efectivo de la población •}

La $\Delta F$ y el $N_{e}$ estimados en las razas de bovinos mexicanos estudiadas se muestran en el Cuadro 6. El $N_{e}$ osciló entre 24 (SA) y 192 (LI). La $\Delta F$ se comportó de forma análoga al $N_{e}$, debido a que son inversamente proporcionales. Según la $\mathrm{FAO}^{(37)}$, para la conservación y mejora de las poblaciones es deseable contar con un $N_{e}$ mínimo de 50, que es equivalente al $1 \%$ en la $\Delta F$. De las poblaciones del presente estudio, sólo dos poblaciones (BR y SA) se encontraron por debajo del nivel recomendado por la FAO. Henson ${ }^{(38)}$ señaló que la heterocigosidad o diversidad genética presente en cada generación decrece a una tasa acelerada en poblaciones con $N_{e}$ menor que 100 y que el incremento en homocigosis como consecuencia de un $N_{e}$ reducido resulta en la pérdida de habilidad adaptativa, depresión endogámica y por último la extinción. Esta situación sería preocupante en cuatro de las razas 
analizadas en este estudio (BN, BR, HE y SA); sin embargo, dado el carácter transfronterizo de las razas estudiadas, es posible la migración y flujo de germoplasma entre países. Bajo este escenario, la entrada de genes nuevos a las poblaciones de bovinos de México, podría traer beneficios a la diversidad genética nacional; sin embargo, de no hacerse, podría afectar negativamente en el caso de diseminación masiva de material genético de pocos reproductores por diversas técnicas reproductivas en animales altamente seleccionados.

Cuadro 6: Tasas de incremento en consanguinidad promedio $(\Delta F)$ y tamaños efectivos de población $\left(N_{e}\right)$ de las siete poblaciones bovinas mexicanas estudiadas, considerando los 10 años más recientes

\begin{tabular}{lccccccc}
\hline & \multicolumn{7}{c}{ Población (raza) } \\
\cline { 2 - 8 } Parámetro & AN & BN & BR & HE & LI & SA & SE \\
\hline$\Delta F$ & 0.32 & 0.74 & 1.04 & 0.65 & 0.26 & 2.08 & 0.45 \\
$N_{e}$ & 155 & 68 & 48 & 77 & 192 & 24 & 110 \\
\hline
\end{tabular}

AN= Angus; BN= Brangus Negro; BR= Brangus Rojo; HE= Hereford; LI= Limousin; SA= Salers; SE= Suizo Europeo.

El $N_{e}$ estimado a partir del incremento en consanguinidad individual ha sido evaluado en pedigríes simulados y reales de distintas especies ${ }^{(39,40,41)}$. Danchin-Burge et $\mathrm{l}^{(40)}$ realizaron una comparación entre tres distintas metodologías utilizadas en la obtención del $N_{e}$ para ocho razas de ganado lechero francés, una mediante la obtención de la $\Delta F$ a partir de la regresión lineal del $F$ sobre el tiempo, otra mediante el cálculo del incremento individual en el $F$, y la otra basada en el incremento en la coancestría. En general, el método de incremento individual en el $F$ presentó valores mayores que los otros dos, donde la menor diferencia fue estimada en las poblaciones más grandes y con mejor calidad de pedigrí. Según Cervantes et $a l^{(39)}$, la $\Delta F$ individual depende en gran parte de la calidad de pedigrí y necesita al menos cinco generaciones completas equivalentes para hacerse constante. Al respecto, se comenta que cuando la información de pedigrí es incompleta, puede provocar sesgos al estimar la $\Delta F$ y, en consecuencia, del $N_{e}^{(12)}$.

Danchin-Burge et al ${ }^{(40)}$ evaluaron el $N_{e}$ a partir del $\Delta F$ individual, obteniendo valores de $N_{e}$ entre 67 y 243 en ocho razas francesas de bovinos lecheros, mientras que en otro trabajo ${ }^{(41)}$ obtuvieron un estimado de 94 en la raza Gir de Brasil. Por otro lado, valores reportados de $N_{e}$ por otros métodos han sido extremadamente variables, donde las razas locales y lecheras especializadas son las que presentan los menores valores ${ }^{(14,23,26,28,42)}$. En general, las poblaciones analizadas en el presente estudio se encontraron dentro del intervalo de la mayoría de valores publicados, excepto SA que tuvo un $N_{e}$ inferior. 


\section{- Probabilidad de origen del gen •}

Los parámetros descriptivos de la probabilidad de origen del gen de las siete poblaciones estudiadas se muestran en el Cuadro 7. En general, las estimaciones de los parámetros relacionados con la probabilidad de origen de los genes indican que la presencia de contribuciones desbalanceadas del uso de los animales fundadores, los cuellos de botella genéticos y la deriva genética, han tenido un efecto importante en las poblaciones actuales (10 años más recientes), con la consecuente pérdida de diversidad genética.

Cuadro 7: Parámetros descriptivos de la probabilidad de origen de los genes estimados en las siete poblaciones bovinas mexicanas estudiadas, para la población total y la población de referencia* (dentro de paréntesis)

\begin{tabular}{lccccccc}
\hline & \multicolumn{7}{c}{ Población total (población de referencia) } \\
\cline { 2 - 8 } Parámetro & AN & BN & BR & HE & LI & SA & SE \\
\hline$f$ & $10,168(9,019)$ & $4,827(3,622)$ & $2,131(1,855)$ & $2,439(1,618)$ & $5,862(4,214)$ & $1,728(1,134)$ & $11,886(3,412)$ \\
$f_{e}$ & $541(431)$ & $113(97)$ & $192(125)$ & $413(308)$ & $519(299)$ & $331(237)$ & $307(246)$ \\
$f_{a}$ & $224(168)$ & $33(27)$ & $55(32)$ & $254(159)$ & $199(96)$ & $105(62)$ & $166(87)$ \\
$f_{a} / f_{e}, \%$ & $41.4(39.0)$ & $29.2(27.8)$ & $28.6(25.6)$ & $61.5(51.6)$ & $38.3(32.1)$ & $31.7(26.2)$ & $54.1(35.4)$ \\
$f_{g}$ & 189 & 19 & 54 & 361 & 187 & 90 & 93 \\
$C_{\text {max }}, \%$ & $3.2(3.4)$ & $13.0(14.6)$ & $9.3(11.9)$ & $1.5(2.3)$ & $2.6(4.2)$ & $4.4(6.3)$ & $3.1(3.8)$ \\
$N_{50}$ & $177(102)$ & $16(12)$ & $44(16)$ & $94(56)$ & $100(190)$ & $44(25)$ & $42(31)$ \\
\hline
\end{tabular}

AN= Angus; BN= Brangus Negro; BR= Brangus Rojo; HE= Hereford; LI= Limousin; SA= Salers; SE= Suizo Europeo.

* Población de referencia (10 años más recientes).

$* f=$ número total de fundadores; $f_{e}=$ número efectivo de fundadores; $f_{a}=$ número efectivo de ancestros;

$C_{\max }=$ contribución del principal ancestro; $N_{50}=$ número de ancestros que contribuyen con el $50 \%$ de los genes a la población; $f_{g}=$ número efectivo de genomas fundadores.

Los valores de los $f$ fueron mayores que los $f_{e}$, lo que indica disminución en diversidad genética, debido a desbalances en el uso de los animales fundadores. En porcentaje, la contribución desigual de los fundadores $\left(f_{e} / f\right)$ en las PT fue más importante en AN, BN y SE (2.3 a $5.3 \%$ ) que en BR, HE, LI y SA (8.9 a $19.2 \%$ ); valores que fueron similares en las respectivas PR, con sólo ligera mejora detectada para SE. La contribución desigual de los fundadores es indicador de posibles incrementos en consanguinidad para generaciones futuras, lo que reflejaría la pérdida de diversidad genética (reducción de heterocigosidad y pocas variantes alélicas por locus) presente en los fundadores ${ }^{(20)}$. En el presente estudio, la contribución muy desbalanceada de los fundadores implica que un reducido número de animales se están utilizando como reproductores en BR, LI y SA, mientras que en ese 
indicador para $\mathrm{HE}$, adicionalmente pudiera tener influencia la poca integridad del pedigrí. Al respecto, Boichard $e t a l^{(12)}$ señalan que en poblaciones con baja calidad de pedigrí puede existir una gran cantidad de fundadores fantasmas, lo que provoca sobreestimaciones del $f \mathrm{y}$ $f_{e}$. Algunas poblaciones de razas de bovinos para carne transfronterizas (Chianina, Marchigniana, Romagnola y Nelore) han mostrado una baja relación (0.23 a $1.09 \%$ ) en los parámetros mencionados ${ }^{(22,43)}$. No obstante, valores similares a los de este estudio han sido reportados en otros estudios con razas bovinas para carne (Charolais, Limousin, Hereford, Angus y Simmental) ${ }^{(23,28)}$.

En el presente estudio, el $f_{a}$ estimado en las siete poblaciones bovinas analizadas fue menor que su respectivo $f_{e}$, lo que indica disminución en la diversidad genética por la presencia de cuellos de botella genéticos en todas las poblaciones, definidas a partir de su fundación. Los efectos del cuello de botella fueron más importantes en los pedigríes de las PT para AN, BN, $\mathrm{BR}, \mathrm{LI}$ y SA $\left(f_{a} / f_{e}<50 \%\right)$, mientras que para HE y SE se estimaron valores mayores que 50 $\%$. Lo anterior podría sugerir un efecto pequeño de los cuellos de botella genéticos en esas últimas poblaciones; sin embargo, la información debe considerarse con cautela en HE, dado que esa población es la que presentó peor integridad de pedigrí. De acuerdo con algunos autores $^{(12)}$, los parámetros derivados de la probabilidad de origen del gen son sensibles a la calidad del pedigrí, aunque en menor grado que el $F$, siendo el $f_{a}$ el más afectado. Los cuellos de botella fueron más estrechos $\left(f_{a} / f_{e}\right.$ entre 26 y $\left.52 \%\right)$ para todas las poblaciones en los 10 años más recientes (PR), lo que indica la necesidad de establecer medidas para incrementar la diversidad genética.

A partir de las contribuciones marginales consideradas en la estimación de los $f_{a}$, es posible identificar a los ancestros más sobresalientes por su contribución marginal relativa a la diversidad genética de la población en estudio ${ }^{(12)}$. Con base en ello, el número de ancestros que explicaron el $50 \%$ de la diversidad genética presente para la PT en cada una de las siete poblaciones estudiadas fue variable, fluctuando desde 16 individuos en BN hasta 177 en AN (Cuadro 7), y con disminuciones para las respectivas PR. Las altas contribuciones marginales de algunos ancestros provocaron que el número de individuos que explican el $50 \%$ de la diversidad genética presente en las poblaciones bajo estudio muestren una relativamente reducida base genética. Diversos autores han reportado cantidades menores que 10 individuos $^{(6,32,40,42)}$, valores menores que en las poblaciones estudiadas en la presente investigación. Sin embargo, estas contribuciones de los ancestros a los genes de las respectivas poblaciones deben ser consideradas por los criadores, debido a que la alta presencia de genes de unos pocos reproductores en las poblaciones podría llevar a un incremento de apareamientos entre animales emparentados, consecuentemente a la generación de individuos consanguíneos y pérdida de variabilidad genética.

En general, $\operatorname{los} f_{\mathrm{a}}$ fueron mayores que $\operatorname{los} f_{g}$ de las $\mathrm{PT}$, sugiriendo disminuciones, de ligeras a moderadas, en la diversidad genética por la presencia de pérdidas aleatorias de alelos en todas 
las poblaciones (deriva genética), desde los progenitores fundadores a sus progenies, por lo que se recomienda el uso de un rango más amplio de sementales.

\section{"Conclusiones e implicaciones \|}

La diversidad genética presente en las poblaciones de bovinos mexicanas evaluadas fue variable. Aunque en todas las poblaciones los coeficientes de consanguinidad promedio fueron relativamente bajos, en algunas se detectaron problemas de pocos reproductores utilizados, tamaño efectivo de población pequeño y potenciales problemas con apareamientos entre individuos emparentados, presentando (en mayor o menor grado) pérdidas en diversidad genética por contribuciones desbalanceadas del uso de los animales fundadores, cuellos de botella genéticos y deriva genética. Considerando los parámetros estudiados, las poblaciones AN, LI y SE fueron las que presentaron la mayor diversidad genética. En SA se requiere incrementar su tamaño efectivo de población, el uso de mayor diversidad en reproductores y la atención en la asignación de apareamientos. Las razas BN, BR y HE presentaron las mayores pérdidas de diversidad genética e incrementos en consanguinidad, por lo que se deben implementar estrategias para mantener su variabilidad genética futura; específicamente en HE se debe mejorar la integridad y completitud de su pedigrí, ya que afecta la estimación de algunos de los parámetros y su interpretación apropiada. A pesar que se detectan esfuerzos recientes de los criadores en el registro de información genealógica de sus animales, se recomienda continuar con el monitoreo de parámetros relacionados con la diversidad genética y la captura de información de pedigrí. Además, aunque se trata de razas transfronterizas, es necesario implementar estrategias para incrementar sus tamaños efectivos, evitar los incrementos en consanguinidad y las pérdidas de diversidad genética, mediante los diseños de apareamientos y el uso estratégico de migración con genes provenientes de otros países. 


\section{Agradecimientos V}

Se agradece a las Asociaciones Mexicanas de Criadores de Ganado: Angus, Brangus, Hereford, Limousin, Salers y Suizo Europeo, por facilitar las bases de datos genealógicos para el presente estudio; y al Consejo Nacional de Ciencia y Tecnología por el financiamiento para los estudios de Maestría del segundo autor.

\section{- Literatura citada:}

1. Santana Jr. ML, Pereira RJ, Bignardi AB, Ayres DR, Menezes GRO, Silva LOC, et al. Structure and genetic diversity of Brazilian Zebu cattle breeds assessed by pedigree analysis. Livest Sci 2016;187:6-15.

2. Sheikhlou M, Abbasi MA. Genetic diversity of Iranian Lori-Bakhtiari sheep assessed by pedigree analysis. Small Ruminant Res 2016;141:99-105.

3. Bernardes PA, Grossi DA, Savegnago RP, Buzanskas ME, Ramos SB, Romanzini EP, et al. Population structure of Tabapuã beef cattle using pedigree analysis. Livest Sci 2016;187:96-101.

4. Abeledo CM, Santana I, Hernández S, Acuña N, Gutiérrez M, Brache JF, et al. Parámetros poblacionales en cerdos CC21 cubanos a partir de su información genealógica. AICA 2017;9:122-128.

5. Barros EA, Brasil LHA, Tejero JP, Delgado-Bermejo JV, Ribeiro MN. Population structure and genetic variability of the Segureña sheep breed through pedigree analysis and inbreeding effects on growth traits. Small Ruminant Res 2017;149:128-133.

6. Ruíz-Flores A, Núñez-Domínguez R, Ramírez-Valverde R, Domínguez-Viveros J, Mendoza-Domínguez M, Martínez-Cuevas E. Niveles y efectos de la consanguinidad en variables de crecimiento y reproductivas en bovinos Tropicarne y Suizo Europeo. Agrociencia 2006;40:289-301.

7. Domínguez-Viveros J, Rodríguez-Almeida FA, Ortega-Gutiérrez JA, SantellanoEstrada E. Análisis de la información genealógica y estimación de parámetros de poblaciones en bovinos de Lidia y equinos de pura raza española de México. Rev Cient FCV-LUZ 2012;22:443-450. 
8. Ramírez-Valverde R, Núñez-Domínguez R, Fabián-Ramos E. Caracterización de las publicaciones sobre mejoramiento genético animal en revistas científicas mexicanas. Ecosist Recur Agropec 2014;1(1):59-72.

9. Belonsky GM, Kennedy BW. Selection on individual phenotype and best linear unbiased predictor of breeding values in a closed swine herd. J Anim Sci 1988;66(5):1124-1131.

10. Ríos UA. Evaluación genética nacional. En: Montaño BM, Martínez VG editores. Guía técnica de control de producción y mejoramiento genético en bovinos para carne. México, DF. Editado por SAGARPA-CONARGEN; 2010:32-44.

11. Gutiérrez JP, Goyache F. A note on Endog: a computer program for analyzing pedigree information. J Anim Breed Genet 2005;122:172-176.

12. Boichard D, Maignel L, Verrier E. The value of using probabilities of gene origin to measure genetic variability in a population. Genet Sel Evol 1997;29:5-23.

13. Boichard D, Maignel L, Verrier E. Analyse généalogique des races bovines laitières françaises. Prod Anim 1996;9(5):323-335.

14. Gutiérrez JP, Altarrabia J, Díaz C, Quintanilla R, Cañón J, Piedrafita J. Pedigree analysis of eight Spanish beef cattle breeds. Genet Sel Evol 2003;35:43-63.

15. Meuwissen THE, Luo Z. Computing inbreeding coefficients in large populations. Genet Sel Evol 1992;24:305-3013.

16. Cervantes N. Estructura genética del caballo de pura raza árabe español y su influencia en razas derivadas: aplicación de nuevas metodologías en el cálculo del tamaño efectivo [tesis doctoral]. Madrid, España. Universidad Complutense de Madrid; 2008.

17. Wright S. Evolution in Mendelian populations. Genetics 1931;16:97-159.

18. Gutiérrez JP, Cervantes I, Molina A, Valera M, Goyache F. Individual increase in inbreeding allows estimating realized effective sizes from pedigrees. Genet Sel Evol 2008;40:359-378.

19. Gutiérrez JP, Cervantes I, Goyache F. Improving the estimation of realized effective population sizes in farm animals. J Anim Breed Genet 2009;126:327-332.

20. Lacy R. Analysis of founder representation in pedigrees: founder equivalents and founder genome equivalents. Zoo Biol 1989;14:565-578.

21. Ballou JD, Lacy RC. Identifying genetically important individuals for management of genetic variation in pedigreed populations. In: Ballou JD, et al, editors. Population 
management for survival and recovery: Analytical methods and strategies in small population management. New York, USA: Columbia University Press; 1995:76-111.

22. Bozzi R, Franci O, Forabosco F, Pugliese C, Crovetti A, Filippini F. Genetic variability in three Italian beef cattle breeds derived from pedigree information. Ital J Anim Sci 2006;5:129-137.

23. Bouquet A, Venot E, Laloë D, Forabosco F, Fogh A, Pabiou T, et al. Genetic structure of the European Charolais and Limousin cattle metapopulations using pedigree analyses. J Anim Sci 2011;89:1719-1730.

24. Martínez RA, García D, Gallego JL, Onofre G, Pérez J, Cañón J. Genetic variability in Colombian Creole cattle populations estimated by pedigree information. J Anim Sci 2008;86:545-552.

25. Peixoto MGCD, Poggian CF, Verneque RS, Egito AA, Carvalho MRS, Penna VM, et al. Genetic basis and inbreeding in the Brazilian Guzerat (Bos indicus) subpopulation selected for milk production. Livest Sci 2010;131:168-174.

26. Maignel L, Boichard D, Verrier E. Genetic variability of French dairy breeds estimated from pedigree information. Interbull Bulletin 1996;14:49-54.

27. Santana Jr. ML, Oliveira PS, Eler JP, Gutiérrez JP, Ferraz JBS. Pedigree analysis and inbreeding depression on growth traits in Brazilian Marchiguiana and Bonsmara breeds. J Anim Sci 2012;90:99-108.

28. Mc Parland S, Kearney JF, Rath M, Berry DP. Inbreeding trends and pedigree analysis of Irish dairy and beef cattle populations. J Anim Sci 2007;85:322-331.

29. Carolino N, Gama LT. Indicators of genetic erosion in an endangered population: The Alentejana cattle breed in Portugal. J Anim Sci 2008;86:47-56.

30. Gebremariam W, Forabosco F, Zumbach B, Palucci V, Jorjani H. Characterization of the global Brown Swiss cattle population structure. Interbull Bulletin 2010;42:16-20.

31. Pirchner F. Population genetics in animal breeding. New York, USA: Plenum Press; 1983.

32. Kadlecík O, Pavlík I. Genealogical analysis in small populations: The case of four Slovak beef cattle breeds. Slovak J Anim Sci 2012;45:111-117.

33. Goyache F, Gutiérrez JP, Fernández I, Gómez E, Álvarez I, Díez J, et al. Using pedigree information to monitor genetic variability of endangered populations: The Xalda sheep breed of Asturias as an example. J Anim Breed Genet 2003;120:95-105. 
34. Ivanković A, Ramljak J, Kelava N, Konjačić M. Pedigree analysis of the Croatian autochthonous cattle breeds: Management of conservation strategy. Stočarstvo 2010;63:3-15.

35. Dunner S, Checa ML, Gutiérrez JP, Martin JP, Cañon J. Genetic analysis and management in small populations: The Asturcon pony as an example. Genet Sel Evol 1998;30:397-405.

36. Verrier E, Colleau JJ, Foulley JL. Long-term effects of selection based on the animal model BLUP in a finite population. Theor Appl Genet 1993;87:446-454.

37. FAO. Secondary guidelines for development of national farm animal genetic resources management plans: Management of small populations at risk. Rome, Italy: FAO; 1998.

38. Henson EL. In-situ conservation of livestock and poultry. Rome, Italy: FAO Animal Production and Health Paper 99; 1992.

39. Cervantes I, Goyache F, Molina A, Valera M, Gutiérrez JP. Application of individual increase in inbreeding to estimate effective sizes from real pedigrees. J Anim Breed Genet 2008;125:301-310.

40. Danchin-Burge C, Leroy G, Brochard M, Moureaux S, Verrier E. Evolution of the genetic variability of eight French dairy cattle breeds assessed by pedigree analysis. J Anim Breed Genet 2012;129:206-217.

41. Santana Jr. ML, Pereira RJ, Bignardi AB, El Faro L, Tonhati H, Albuquerque LG. History, structure, and genetic diversity of Brazilian Gir cattle. Livest Sci 2014;163:2633.

42. Sorensen AC, Sorensen MK, Berg P. Inbreeding in Danish dairy cattle breeds. J Dairy Sci 2005;88:1865-1872.

43. Vozzi PA, Marcondes CR, Magnabosco CU, Bezerra LAF, Lobo RB. Structure and genetic variability in Nellore (Bos indicus) cattle by pedigree analysis. Genet Mol Biol 2006;29:482-485. 